\title{
La edad como factor de éxito en las relaciones de parejas casadas
}

Ruth Berenice Aldana Zamorano ${ }^{1}$ y Gabriela Eugenia López Tolsa Gómez ${ }^{1}$

\section{Introducción}

Se ha planteado que la satisfacción marital juega un papel importante en la relación ya que ésta es producto de una evaluación global de diversos aspectos sobre la relación de pareja (Díaz Loving y Sánchez Aragón, 2002). En el presente trabajo se pretende investigar en qué medida la edad llega a influir para alcanzar el éxito personal y, como consecuencia, la satisfacción en la relación de pareja.

Por su parte, "el éxito es una actitud intencional ante la vida que garantiza ser un administrador fiel de dones y talentos. Te obliga a crecer, desarrollarte y fuerza a abandonar la zona de comodidad; no depende de las situaciones externas de la vida, sino de la condición interna propia" (Newberry, 2007).

En varias investigaciones se han tomado en cuenta diferentes factores para evaluar una relación de pareja, como el contexto cultural en el que los individuos se desenvuelven (Mureira, 2011), si existe o no maltrato físico o psicológico (Sarasua,

$1 \quad$ Universidad Internacional. 
Zubizarreta y Echeburúa y Corral, 2007), la realización de ambos miembros de la pareja (Acevedo y Restrepo, 2010), el lugar de residencia, el sexo, el estatus de pareja (García y Reyes, 2009) y los estilos de apego (Guzmán y Contreras, 2012). Se ha planteado que cada uno de esos factores guarda relación con el hecho de que un matrimonio se sienta satisfecho con su relación; sin embargo, a pesar de que se menciona la edad de los participantes y es posible sacar conclusiones con base en la relación que se da entre la edad y esos factores, no existe un estudio que evidencie de forma directa a ésta como el factor principal que sea capaz de proporcionar el éxito personal del individuo y que, por tanto, llegue a modificar la manera en que cada uno de los miembros se siente satisfecho con su relación. Por ello, el objetivo del presente fue analizar el papel que desempeña la edad para poder alcanzar el éxito personal y de esta forma conocer si este factor influye en la satisfacción con la pareja.

\section{Método}

\section{Participantes}

Participaron 12 matrimonios heterosexuales (civil o religioso) de la ciudad de Guadalajara, divididos en dos grupos de acuerdo al promedio de edad de ambos integrantes: parejas jóvenes (20 a 29 años; 27.4 de promedio) y parejas maduras (31 a 40 años; 35.3 de promedio).

\section{Instrumento}

Se utilizó un cuestionario diseñado ex profeso, conformado por 20 preguntas de opción múltiple en forma de escala de Likert, que pretendían evaluar las siguientes áreas: nivel de estudios, área laboral, pareja en general, motivos de las discusiones, consecuencias de las mismas y crecimiento personal en relación con el cónyuge. Cada pregunta tenía un valor máximo de 5\%, obteniéndose así el valor total asignado por área. A cada inciso 
se le asignó un valor entre 0 y 5 , de forma que a mayor puntaje obtenido, menor nivel de éxito personal y, por tanto, menor probabilidad de satisfacción en la pareja.

\section{Procedimiento}

Los cuestionarios se aplicaron de forma individual para que las respuestas de cada participante no fueran influidas por las de su pareja. Después se calificó cada prueba y se prepararon los datos para el análisis.

\section{Resultados}

Al inicio del análisis se obtuvo el promedio de puntaje por grupos de edad. Para las parejas maduras, el puntaje en la prueba de satisfacción fue de 19.16 y para las jóvenes, de 27.83; esto indica que las parejas jóvenes tienen menor probabilidad de alcanzar la satisfacción en pareja.

De la misma forma, se calculó la diferencia entre los puntajes de ambos miembros del matrimonio y se observó que en cuatro de las seis parejas maduras la mujer fue quien mostró mayores niveles de satisfacción (menores puntajes). En las parejas jóvenes se observó lo contrario, ya que en cuatro de seis parejas fue el hombre quien mostró los mayores niveles. Al comparar el promedio de la diferencia en los puntajes, se observó que la diferencia es mayor en las parejas maduras que en las parejas jóvenes, lo cual quiere decir que a pesar de ser las parejas jóvenes quienes están menos satisfechas con su relación, son las más congruentes al momento de contestar.

Una situación que se observó en ambos tipos de pareja fue que el integrante con mayor edad dentro de la relación fue quien obtuvo puntajes menores, es decir, tiene mayor éxito a nivel personal y, por tanto, reporta sentirse más satisfecho con su relación de pareja. En once de las doce parejas analizadas, el hombre fue mayor que la mujer. También se observó que no existe relación entre la diferencia de edad y la diferencia de puntajes. 
Asimismo, se calculó el promedio por áreas de ambos tipos de parejas. Al compararlos, se pudo observar que tanto en parejas jóvenes como maduras el área mayormente afectada fue la de "Pareja en general". Asimismo, el "area laboral" ocupó el mismo lugar en cuanto nivel de afectación en los dos tipos de pareja. En las parejas jóvenes "el crecimiento personal en relación con el esposo(a)" es de las áreas más afectadas, mientras que en las parejas maduras ocurre lo contrario, ocupando uno de los últimos lugares en cuanto a nivel de gravedad. Por último, las áreas referentes a "motivos" y "consecuencias de las discusiones", en ambos tipos de pareja son las menos afectadas. Las parejas jóvenes obtuvieron promedios superiores en todas las áreas a excepción de la de "nivel de estudios".

\section{Discusión}

De acuerdo a los datos antes mencionados, se pudo observar cómo el éxito personal alcanzado con la edad parece influir en la satisfacción con la relación de pareja.

Los resultados de este estudio coinciden con otras investigaciones, como es el caso de Sarasua et al. (2007), quienes a pesar de haberse dedicado a analizar específicamente la existencia de maltrato físico y psicológico, llegaron a la conclusión de que las parejas jóvenes son más propensas a que se presente alguno de los anteriores, lo cual habla de que en las parejas con edades más avanzadas es más probable que lleguen a sentirse satisfechas con su relación, como se ha reportado aquí.

De igual forma, los datos recabados coinciden con lo reportado por Acevedo y Restrepo (2010), quienes se dedicaron a analizar las experiencias sobre vivir feliz en pareja, ya que encontraron que los participantes que se sentían más realizados de forma personal tendían a evaluar mejor su relación de pareja. Los que contestaron de tal forma fueron los participantes de edades más avanzadas, los cuales dan referencia de estar satisfechos consigo mismos y, por lo tanto, se desempeñan mejor en su relación. 
García y Reyes (2009), por su parte, al investigar sobre las actitudes hacia el matrimonio y el divorcio, hicieron énfasis en el sexo, la residencia y el estatus de pareja, pero no consideraron la edad como factor principal en su estudio; sin embargo, encontraron que los jóvenes actualmente no buscan compromisos para así evitar futuros divorcios. Lo anterior podría explicar por qué las parejas jóvenes parecen sentirse menos satisfechas con su relación que las parejas maduras.

Comparando la presente investigación con lo reportado por Martínez, Rodríguez y Moya (2010), quienes se centraron en saber por qué tienen éxito y fracasan las personas con poder y sin poder, se encontró que estos autores afirman que el poder otorga el éxito o fracaso a nivel personal, lo cual es correcto; sin embargo, hace falta considerar la edad, pues con ésta aumenta la probabilidad de alcanzarlo. Relacionándolo con el presente, lo anterior influirá en la manera en que hombre y mujer actúan dentro de la relación.

Además, en el estudio realizado por Guzmán y Contreras (2012), quienes se dedicaron a analizar los estilos de apego en relaciones de pareja y su asociación con la satisfacción marital, le atribuyeron una buena relación de pareja al tipo de apego existente entre los miembros, siendo el seguro el más propenso a generar la satisfacción marital. Lo anterior es importante debido a que se considera que el tipo de apego puede variar conforme a la edad y el éxito personal alcanzado por la misma.

Un dato interesante y que no se consideró en otras investigaciones que han estudiado la manera de llevar una relación, es que hombres y mujeres respondieron diferente el cuestionario. En las parejas jóvenes, el hombre tiende a ser el más cercano a sentirse satisfecho con su relación, mientras que en las maduras fue la mujer en su mayoría la que lo reportó. Esto puede deberse a que los hombres tienden a comenzar a laborar desde más jóvenes, adquiriendo de esta forma una realización más temprana, quienes a pesar de no contar con un mayor nivel de estudios, se sienten satisfechos y aprovechan las habilidades con las que ya cuentan. Por su lado, es probable que las mujeres tiendan a esperar en búsqueda de empleos más formales o simplemente 
le atribuyan sentirse realizadas a otros aspectos como ya contar con su propia familia.

Otros datos importantes que no han sido considerados son las áreas que se analizaron, ya que en otras investigaciones se consideraban algunas como el nivel de estudios, el área laboral, el crecimiento personal o la pareja en general; sin embargo, no se habían contemplado los motivos de las principales discusiones, las cuales suelen ser por problemas de tipo económico. Ambos tipos de pareja señalaron que los motivos económicos son el principal motivo por el cual discuten, aunque las parejas jóvenes suelen hacerlo en un mayor número de ocasiones. Lo anterior guarda relación con el nivel académico y el desempeño en el área laboral de los miembros de la pareja, pues quienes obtuvieron resultados deficientes en estas áreas fueron los mismos que presentaron problemas de tipo económico. Por su lado, en las consecuencias de las discusiones, lo más común tanto para parejas jóvenes como maduras es gritarse, aunque se dieron dos casos en los que sí se llegó a los golpes, esto sucedió únicamente en las parejas jóvenes, de forma similar a lo reportado en la literatura (Sarasua et al, 2007); mientras que en las parejas maduras hubo tres sujetos que respondieron haber pensado incluso en el divorcio.

En conclusión, lo encontrado en este estudio sugirió que el éxito personal es algo que puede llegar a determinarse por la edad y que influye en que una pareja llegue a sentirse satisfecha con su relación; sin embargo, será importante realizar un estudio con una muestra más amplia, así como un mayor rango de edad, para verificar la generalidad del fenómeno, ya que a pesar de haberse visto reflejado en los participantes, debe considerarse lo anterior para poder tener un mayor nivel de confiabilidad en los datos reportados.

\section{Referencias}

Acevedo, V. y Restrepo, L. (2010). Experiencias de parejas sobre vivir feliz en pareja. Pensamiento Psicológico, 8(15), 63-76. 
Díaz-Loving, R. y Sánchez Aragón, R. (2002). Psicología del amor: Una visión integral de la relación de pareja. México: Editorial Porrúa.

García, M. y Reyes I. (2009). Actitudes hacia el matrimonio y el divorcio: diferencias por sexo, residencia y estatus de pareja. Psicología Iberoamericana, 17(1), 48-56.

Guzmán, M. y Contreras, P. (2012). Estilos de apego en relaciones de pareja y su asociación con la satisfacción marital. Psikhe, 21(9), 69-82.

Martínez, R., Rodríguez, R. y Moya, M. (2010). ¿Por qué tienen éxito y fracasan las personas con poder y sin poder? Poder y atribuciones de control. Universitas Psychologica, 9(1), 57-66.

Mureira, F. (2011). Los cuatro componentes de la relación de pareja. Revista Electrónica de Psicología Iztacala, 14(1), 321-332.

Newberry, T. (2007). El éxito no es casualidad. Estados Unidos: Tyndalehouse.

Sarasua, B., Zubizarreta E., Echeburúa, E. y Corral, P. (2007). Perfil psicopatológico diferencial de las víctimas de violencia de pareja en función de la edad. Psicothema, 19(3), 459-460. 
